\title{
Temporal and spatial dynamics in the apple flower microbiome in the presence of the phytopathogen Erwinia amylovora
}

\author{
Zhouqi Cui ${ }^{1} \cdot$ Regan B. Huntley ${ }^{1} \cdot$ Quan Zeng $\mathbb{D}^{1} \cdot$ Blaire Steven $\mathbb{( D}^{2}$
}

Received: 15 January 2020 / Revised: 9 September 2020 / Accepted: 17 September 2020 / Published online: 6 October 2020

(c) The Author(s), under exclusive licence to International Society for Microbial Ecology 2020

\begin{abstract}
Plant microbiomes have important roles in plant health and productivity. However, despite flowers being directly linked to reproductive outcomes, little is known about the microbiomes of flowers and their potential interaction with pathogen infection. Here, we investigated the temporal spatial dynamics of the apple stigma microbiome when challenged with a phytopathogen Erwinia amylovora, the causal agent of fire blight disease. We profiled the microbiome from the stigmas of individual flowers, greatly increasing the resolution at which we can characterize shifts in the composition of the microbiome. Individual flowers harbored unique microbiomes at the operational taxonomic unit level. However, taxonomic analysis of community succession showed a population gradually dominated by bacteria within the families Enterobacteriaceae and Pseudomonadaceae. Flowers inoculated with E. amylovora established large populations of the phytopathogen, with pathogen-specific gene counts of $>3.0 \times 10^{7}$ in $90 \%$ of the flowers. Yet, only $42 \%$ of inoculated flowers later developed fire blight symptoms. This reveals that pathogen abundance on the stigma is not sufficient to predict disease outcome. Our data demonstrate that apple flowers represent an excellent model in which to characterize how plant microbiomes establish, develop, and correlate with biological processes such as disease progression in an experimentally tractable plant organ.
\end{abstract}

\section{Introduction}

The most important function of flowers, the reproductive organs of angiosperms, is to provide a mechanism for pollination, the union of sperm contained within pollen, to the ovules contained in the ovary. The fertilized ovules produce seeds that will later germinate to become the next generation plants. Yet, unlike other vegetative organs, such as the roots, stems, and leaves that are present through a

Supplementary information The online version of this article (https:// doi.org/10.1038/s41396-020-00784-y) contains supplementary material, which is available to authorized users.

Quan Zeng

quan.zeng@ct.gov

$\triangle$ Blaire Steven

blaire.steven@ct.gov

1 Department of Plant Pathology and Ecology, The Connecticut Agricultural Experiment Station, New Haven, CT 06511, USA

2 Department of Environmental Sciences, The Connecticut Agricultural Experiment Station, New Haven, CT 06511, USA large part of the plant's lifecycle, flowers develop on mature plants and are typically present for the limited period during bloom. As such, research characterizing the microbiome of the flower is generally less developed than for other plant organs.

Flowers of apple, also known as apple blossoms, (Malus $x$ domestica) have been subject to considerable research attention as they are the direct precursors of apple fruits, one of the most consumed fruits worldwide [1]. The ephemeral nature of apple flowers, with mature flowers from opening of the blossom to petal fall only lasting for 5-10 days in spring, offers a unique environment in which to study community succession [1,2]. During bloom, petals open in a relatively short period of time, typically within 1 day, which exposes the internal flower parts to the environment and microorganisms. Several of these internal flower parts exude various types of nutrient-rich secretions including nectar, stigmatic exudate, and pollen exudate, for the purpose of attracting pollinators, and inducing the germination of pollen grains $[1,3]$. These secretions are rich in sugars, amino acids, polysaccharides, and glycoproteins, which are excellent sources of nutrients for many microorganisms [1, 3, 4]. The stigma is particularly 
nutrient rich and harbors a larger microbial biomass than other flower parts [5, 6]. Previous research has documented a relatively low diversity of the stigma microbiome, although certain lineages predominantly within the families Enterobacteriaceae and Pseudomonadaceae tend to be dominant [7].

While the stigma provides an excellent niche for microbial colonization, it also offers an opportunity for pathogen infection. Many pathogens have evolved to take advantage of this environmental niche, among which one of the most important is the phytopathogenic bacterium Erwinia amylovora $(E a)$, the causal agent of fire blight. Fire blight is considered as one of the most devastating diseases of apple, with annual losses and costs of control estimated at over $\$ 100$ million in the USA [8]. During bloom, $E a$ cells are spread to apple flowers by insects, wind, or rain and multiply on the stigma surface [9]. $E a$ cells can then migrate from the stigma to the hypanthium and enter into the host through natural openings. Initial infection occurs at the ovary tissue and can spread to other parts of the plants through the plant vasculature system. Fire blight infection can result in significant yield reduction and/or tree death. In this regard, uncovering environmental or biologic factors that can inhibit the spread or development of fire blight are of considerable research interest.

One potential source of fire blight control is the natural microbiome of the stigma. Yet, there exist considerable knowledge gaps concerning how the stigma microbiome is established and structured. The studies that have considered the stigma microbiome have generally focused on cataloging microbial diversity through various culturedependent and culture-independent methods [7, 10] and few studies have investigated the temporal development of the microbiome [2]. Furthermore, previous research has predominantly studied the microbiome using pooled flower samples, thus the extent to which the microbiome differs among individual flowers of the same genetic background is uncertain. Finally, how the colonization of a phytopathogen affects the development, composition, or structure of the stigma microbiome is essentially unknown. In this study, we examined the temporal development of the stigma microbiome in the presence and absence of $E a$ to investigate how this organism influences the development of the normal microflora of the apple flower stigma. In addition, we characterized the variability of the microbiome amongst 100 individual stigmas inoculated with $E a$ to assess if the presence and abundance of a pathogen would alter the state of the microbiome, and if any microbiome traits were associated with the proportion of flowers that later developed disease.

\section{Materials and methods}

\section{Sampling site}

To limit the effects of host and environmental conditions, we used flowers from nine trees of the same apple cultivar 'Early Macoun' (Malus pumila NY75414-1) planted at the same geographical location (Lockwood Farm, Hamden, Connecticut, $41.406 \mathrm{~N} 72.906 \mathrm{~W}$ ). All trees were the same age and under the same maintenance program. Weather data (temperature and humidity) prior to and during bloom (from April 29 to May 28, 2018) is summarized in Table S1.

\section{Experiment design and stigma collection}

\section{Labeling flower clusters}

On May 6, 2018, flower clusters that were in "king bloom" stage (central flower opened but the six side flowers still closed, see Fig. 1a) were labeled with plastic tags. Flower clusters were located randomly on the trees to maximize spatial variation. The day after the flower clusters were tagged (May 7), we identified the tagged clusters in which all the side flowers were open, and flower clusters with unopened side flowers were not used. This defined the Day 1 flowers. In this manner, only side flowers of roughly the same developmental stage, the first day of petal opening, were used in subsequent experiments.

\section{Sampling for temporal alterations of the stigma microbiome}

On May 7 , ten of the $\sim 40$ tagged flower clusters were selected, and the stigmas of an individual side flower collected from a unique flower cluster were harvested with sterile scissors (see Fig. 1b) and placed in a sterile $1.5 \mathrm{ml}$ microcentrifuge tube. Collected stigma samples were kept in liquid nitrogen and transported to the laboratory for immediate processing. These samples were labeled as day 1 samples. The next day, another ten flowers were selected for DNA extraction as described above (as day two samples). Immediately after sample collection on day 2, $E a$ was inoculated onto 20 tagged flower clusters and labeled as $E a$ treated. The inoculum consisted of an overnight culture of $E a 110$ grown in lysogeny broth diluted to a final concentration of $1 \times 10^{6} \mathrm{CFU} / \mathrm{ml}$ in sterile water. The diluted culture was spray-inoculated to the open flowers using a handheld sprayer to ensure every flower was evenly exposed. Another 20 flower clusters were sprayed with sterile water as water controls. On each day from day 3 to day 5, stigmas from $20 E a$-treated and 20 watertreated flowers were collected individually from each 

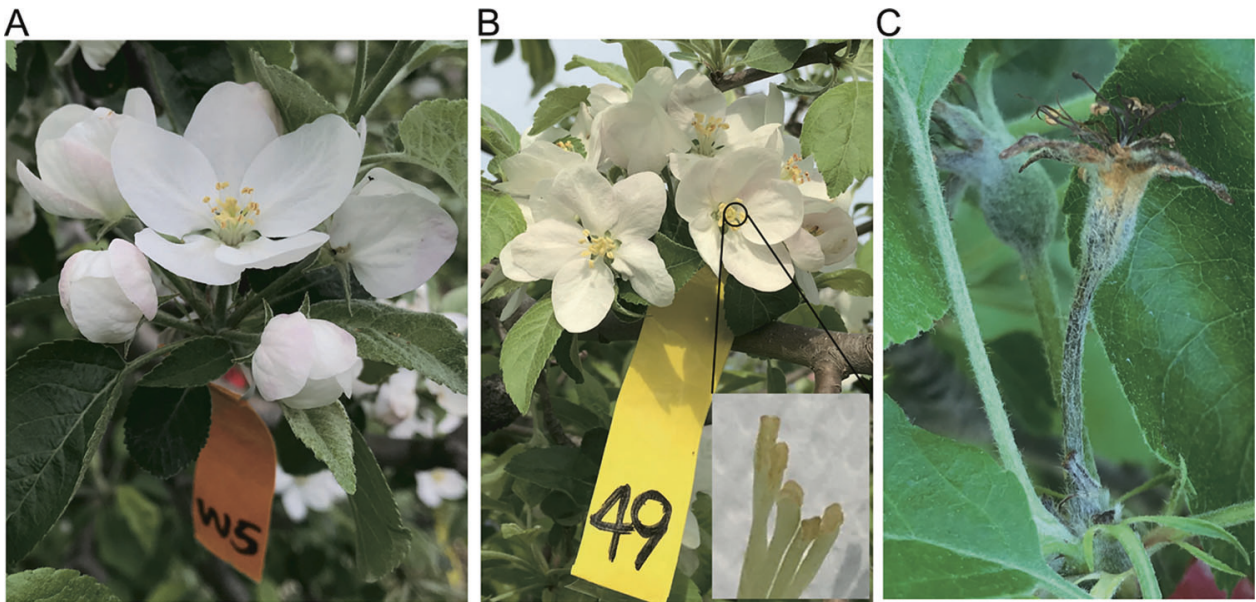

Fig. 1 Illustration of apple flower clusters. a An apple flower cluster at "king bloom." This is when flower clusters of the same age were tagged. b Once the surrounding flowers opened (1 day after king bloom), stigmas of side flowers were sampled and named as "day 1". Each sample contains stigmas collected from an individual

flower cluster and processed according to the same method described above.

\section{Sampling for spatial patterns in the stigma microbiome}

To investigate a larger spatial sampling of Ea-inoculated flowers, we performed a parallel experiment, and tagged an additional 150 flower clusters to ensure flowers used in the experiment were the same age as the rest of the experimental set. As described for the temporal sampling, the flower clusters were individually spray-inoculated with $E a$ $\left(1 \times 10^{6} \mathrm{CFU} / \mathrm{ml}\right)$ on day 2 , and stigma samples of individual flowers were harvested on day 4. A total of 100 flowers of the same developmental stage were harvested for DNA extraction, while the remaining flowers of each flower cluster were left on the tree to monitor disease development. Blossom blight symptoms of black withering and dying of the remaining flowers were evaluated 2 weeks after inoculation on May 24, 2018 (Fig. 1c). An illustrated scheme of both temporal and spatial sampling is shown in Fig. S1.

\section{DNA extraction and sequencing of 16S rRNA genes}

For extraction of bacterial DNA, $200 \mu$ l of $0.5 \times$ phosphatebuffered saline was added to each microcentrifuge tube containing stigma samples. Epiphytic microbes were removed from the stigma by a 5-min water bath sonication followed by a 30-s vortex. DNA was extracted from the $200 \mu \mathrm{l}$ of bacterial suspension by using the DNeasy PowerSoil Kit (Qiagen, Hilden, Germany) according to the manufacturer's instructions. The amount of template DNA added in the PCR reaction $(25 \mu \mathrm{l})$ ranged from $10.0 \mathrm{ng}$ to flower collected from a unique flower cluster. A close up photo of individual stigmas is shown in the inset. c An example showing a flower with fire blight disease (foreground) and a healthy flower (background) coexisting in the same flower cluster. A schematic diagram outlining the sampling design is shown in Fig. S1.

$20.0 \mathrm{ng}$ as determined by Nanodrop2000 (Thermo Fisher Scientific, Waltham, MA). DNA was amplified by using the 515f/806r primer set, which targets the V4 region of the bacterial 16S rRNA gene, with both primers containing a 6bp barcode unique to each sample [11]. PNA clamps were added to the PCR mixture at a concentration of $0.75 \mu \mathrm{M}$ to block the PCR amplification of apple plastid and mitochondrial sequences [7]. PCR conditions were performed as described in Steven et al. [7]. Successful PCR amplifications at the correct amplicon size were confirmed by gel electrophoresis. Negative controls were included for both sequence datasets. Negative controls consisted of sterile water used as a DNA extraction control and subsequent template for PCR. No amplicon bands were ever observed in gel electrophoresis of negative reactions. The PCR products (including negative controls) were purified and normalized by using the SequalPrep normalization plate kit (Invitrogen, CA, USA). Sequencing was conducted on an Illumina MiSeq v2.2.0 platform through services provided by the UConn MARS facility.

\section{Quantitative PCR for enumeration of Ea}

The abundance of $E a$ in each collected stigma sample was quantified by determining the cycle threshold (CT) value of the $E a$-specific gene $a m s C$ [12]. Quantitative PCR (qPCR) was performed using a SsoAdvanced universal SYBR Green supermix (Bio-Rad, CA, USA), as described previously [13]. The CT values for a $1 / 10$ dilution series of known ams $C$ gene copies of $E a$ chromosomal DNA was determined to make a standard curve for calculation of copy numbers in stigma samples. 


\section{Bioinformatics and statistical analysis}

Illumina sequencing reads were assembled into contigs and quality screened by using mothur v1.39.5 as previously described [14]. Sequences that were at least 253 bp in length, contained no ambiguous bases, and no homopolymers of more than $8 \mathrm{bp}$ were used in the analysis. Chimeric sequences were identified by using VSEARCH as implemented in mothur [15], and all potentially chimeric sequences were removed. To maintain a similar sampling effort between samples, samples with less than 10,000 sequences per sample were also removed. The resulting sequence counts per sample are presented in Table S2. Negative control samples did show some sequence recovery but were in the range of 66-341 sequences (Table S2), or less than $1 \%$ of sequences recovered in the sample libraries.

Sampling effort was normalized to the depth of the smallest sample, and operational taxonomic units (OTUs) were defined at $100 \%$ sequence identity, employing the OptiClust algorithm in mothur [16]. Taxonomic classification of sequences was performed with the Ribosomal Database Project classifier against the SILVA v132 reference alignment in mothur $[17,18]$. Non-metric multidimensional scaling (NMDS) was used to visualize the pairwise distances among samples with Bray-Curtis distances in the Vegan package in $\mathrm{R}$ [19]. Descriptive diversity statistics were calculated in mothur. The correlation between alpha diversity determined with the non-parametric Shannon's Diversity Index, and $E a$ abundance in each sample was generated with the ggplot2.0 package for R [20]. Statistically significant differences in diversity metrics were identified with a one-way ANOVA and Tukey-Kramer post hoc test in the agricolae package in $\mathrm{R}$.

\section{Results}

\section{Temporal patterns in stigma microbial community assembly}

We characterized the microbial community on stigmas collected from individual flowers, over a period of 5 days after petal opening, to investigate the temporal dynamics in community assembly and microbial succession on the stigma. Meanwhile, we included $E a$-inoculated stigmas to compare community succession in the presence of a phytopathogen. A total of 2,930,231 high-quality sequences were obtained from 96 samples with the number of sequences ranging from 10,210 to 97,668 (Table S2). These sequences clustered into 46,809 OTUs (mean 222 per sample) at $100 \%$ sequence similarity.

At the phylum level, 24 phyla were detected. In both the water control and $E a$-inoculated datasets, the dominant phylum was Proteobacteria ( $94.3 \%$ of total sequences), followed by Cyanobacteria (3.6\%), Actinobacteria (0.8\%), Firmicutes $(0.2 \%)$, and Bacteroidetes $(0.2 \%)$. A temporal pattern was observed, in that phyla outside the Proteobacteria were most abundant in the early time points (days 1 and 2) accounting for $15 \%$ of sequences and decreasing to $<1 \%$ at later time points (Fig. S2).

Given the dominance of Proteobacteria, these sequences were classified to deeper taxonomic ranks. Sixty-seven families were identified, with the majority of sequences belonging to the Enterobacteriaceae the family to which Erwinia belongs (average 70.0\%, blue bars) and Pseudomonadaceae (26.2\%, red bars in Fig. 2), with small contributions from Moraxellaceae $(0.6 \%)$, Beijerinckiaceae $(0.2 \%)$, unclassified Gammaproteobacteria (0.3\%), Burkholderiaceae $(0.3 \%)$, and Xanthomonadaceae (0.2\%) (Fig. 2). Temporal patterns in the dataset showed that the mean relative abundance of Enterobacteriacea-related sequences was generally stable for the water controls with no significant differences between time points (Fig. 3a). In contrast, the $E a$-treated flowers showed reduced Enterobacteriacea relative abundance on day 2 (Fig. 3a, $P<0.01$ ). For the Pseudomonadaceae populations, there were no significant differences in relative abundance associated with the temporal sampling (Fig. 3b). In regards to differences between the control and $E a$-treated flowers, by day 5 the water controls harbored significantly lower populations of the Enterobacteriacea and significantly higher populations of the Pseudomonadaceae (Fig. 3a, b, $P<0.01$ ). Taken together, these data show that two families of bacteria tend to monopolize the stigma environment and that the $E a$ treatment has a significant effect of the final composition of these populations.

\section{Abundance of Ea on individual flowers}

We employed two methods to assess the abundance of $E a$ in the datasets, relative abundance of $E a$ sequences in the dataset and $E a$ copy numbers quantified by qPCR of an $E a$ specific gene. To estimate $E a$ relative abundance, we identified an OTU in the dataset that had $100 \%$ sequence identity with the inoculated $E a$ strain (OTU1; Table S3). It is important to note that this is only a presumptive identification of $E a$ as the genus Erwinia contains several nonpathogenic strains that may not be distinguished with the short 16S rRNA gene sequence employed for OTU binning in this study [21-23].

OTU1 was detected every day, but not in all samples. On days 1 and 2, prior to the stigma treatments, OTU1 made up an average of $4 \%$ and $6 \%$ of the microbiome sequences, respectively (filled bars in Fig. 2). In the control water sprayed stigmas the proportion of OTU1 gradually increased from an average of $2 \%$ of sequences on day 3 to $13 \%$ on day 4 , finally making up an average of $24 \%$ of 


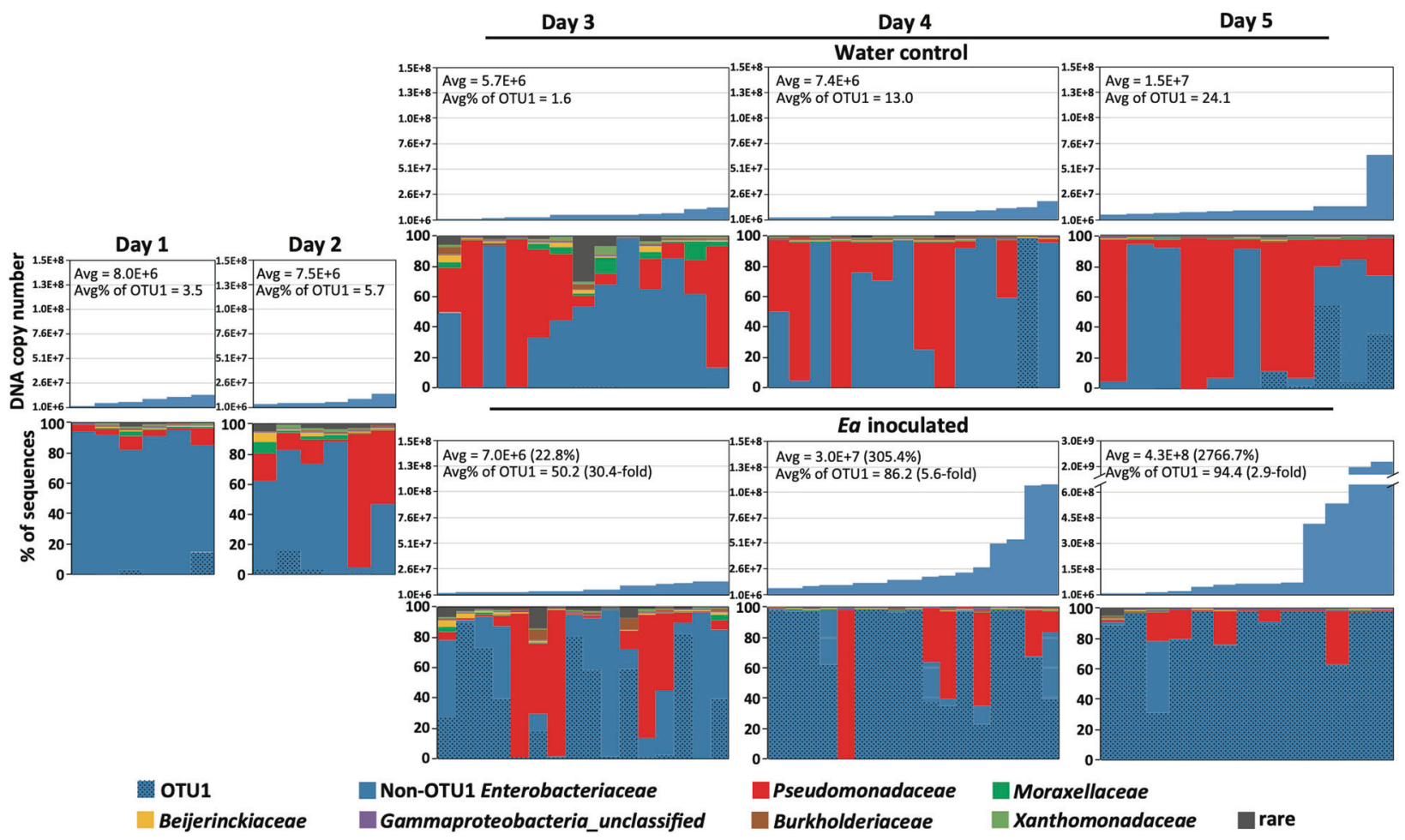

Fig. 2 Temporal dynamics in the predominant bacterial families present on stigmas of individual flowers. Each column represents a single flower. The seven most abundant families are displayed, and the category "rare" represents the sum of the remaining taxa. The relative abundance of OTU1, identified as sharing $100 \%$ sequence identity with Erwinia amylovora, is indicated by hatched lines. Copy numbers of the E. amylovora amsC gene in each sample were determined by

qPCR, and are displayed in the bar graphs above the stacked columns. The average DNA copies are indicated as well as the average relative abundance of OTU1. The change in Ea inoculated compared to water control is labeled in the brackets. Water control: flower clusters sprayed with sterile $\mathrm{H}_{2} \mathrm{O}$. Ea inoculated: flower clusters sprayed with a bacterial suspension of E. amylovora strain 110. Days 1-5 represent the number of days after petals opened during bloom.

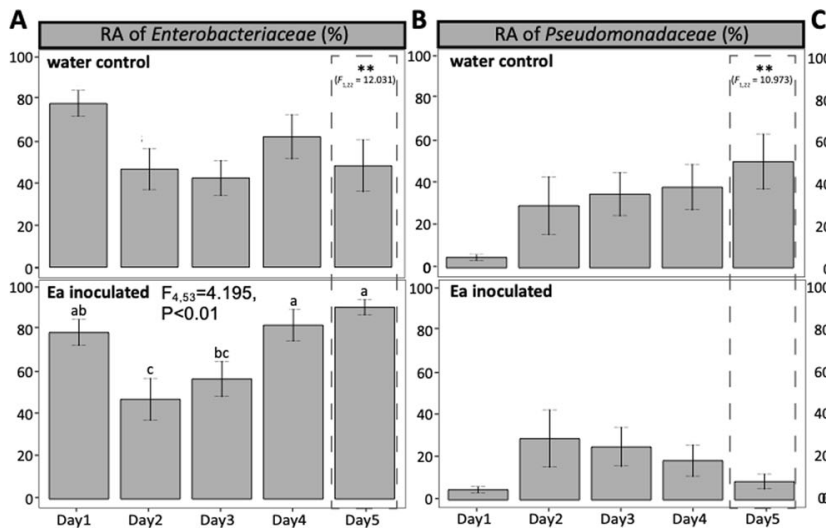

Fig. 3 Comparison of metrics of microbiome composition between water control and $\boldsymbol{E} \boldsymbol{a}$-inoculated flowers. a Relative abundance of Enterobacteriaceae-related sequences. b Relative abundance of Pseudomonadaceae-related sequences. c Relative abundance of OTU1; $100 \%$ sequence identity to $E a$. $\mathbf{d} E a$ ams $C$ gene copy numbers. Within each group, bars labeled with different letters were significantly

sequence on day 5; although the differences were not statistically significant (Fig. 3c). In contrast, the populations of OTU1 were larger in the $E a$-treated stigmas. By day 3 (one day after inoculation) OTU1 accounted for an average of $50 \%$ of the sequence libraries, increasing to $86 \%$ on day 4

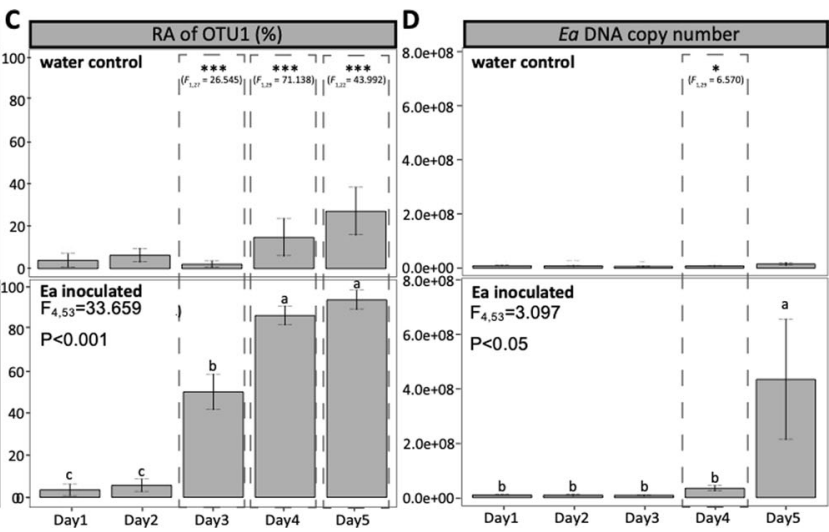

different by one-way ANOVA followed by a post hoc Tukey-Kramer test. The F-statistic and $P$ value of the ANOVA test are indicated. The dashed boxes indicate significant differences between water control and $E a$-inoculated flowers sampled on the same day. The asterisks indicate $P$ values of the test statistic; $* P<0.05$; $* * P<0.01$; $* * * P<$ 0.001. The F-statistic is also indicated within the box.

and ending at $94 \%$ of sequences on day 5 , a 2.9 -fold increase and significantly larger population in comparison to the controls (Fig. 3c, $P<0.001$ ).

In addition, qPCR was performed to quantify the genome copies of $E a$ in each stigma sample. As was observed for 
relative abundance of OTU1, Ea was identified across the dataset. In the pretreated stigmas (days 1 and 2) the average copy number of $E a$ DNA was $8.0 \times 10^{6}$ and $7.56 \times 10^{6}$, respectively (Figs. 2 and $3 \mathrm{~d}$ ). In the control datasets, average $E a$ counts did not significantly increase (Fig. 3d). In the $E a$ inoculated flowers the copy number of $E a$ on day 3 was similar to the control flowers, suggesting $E a$ had not yet established strong growth on the stigma (Figs. 2 and 3d). However, by day 4 the average abundance of $E a$ on the treated stigmas reached $3.0 \times 10^{7}$, a threefold increase compared to the controls, and increased further on day 5 reaching an average of $4.3 \times 10^{8}$, a 28 -fold increase in comparison to the controls (Figs. 2 and 3d). Taken together, these data suggest that $E a$ may be naturally present in an orchard with a history of fire blight infection, as it was commonly detected in the pretreated and control stigmas. Yet, the Ea inoculation clearly increased $E a$ colonization, which was readily apparent by day 4, 2 days after the inoculation (Fig. 3d).

\section{Effects of Ea inoculation on community composition and diversity}

To test if Ea treatment had a significant effect on microbiome composition, we visualized the Bray-Curtis distances among samples of each dataset using NMDS. The samples clearly clustered due to $E a$ inoculation, which was

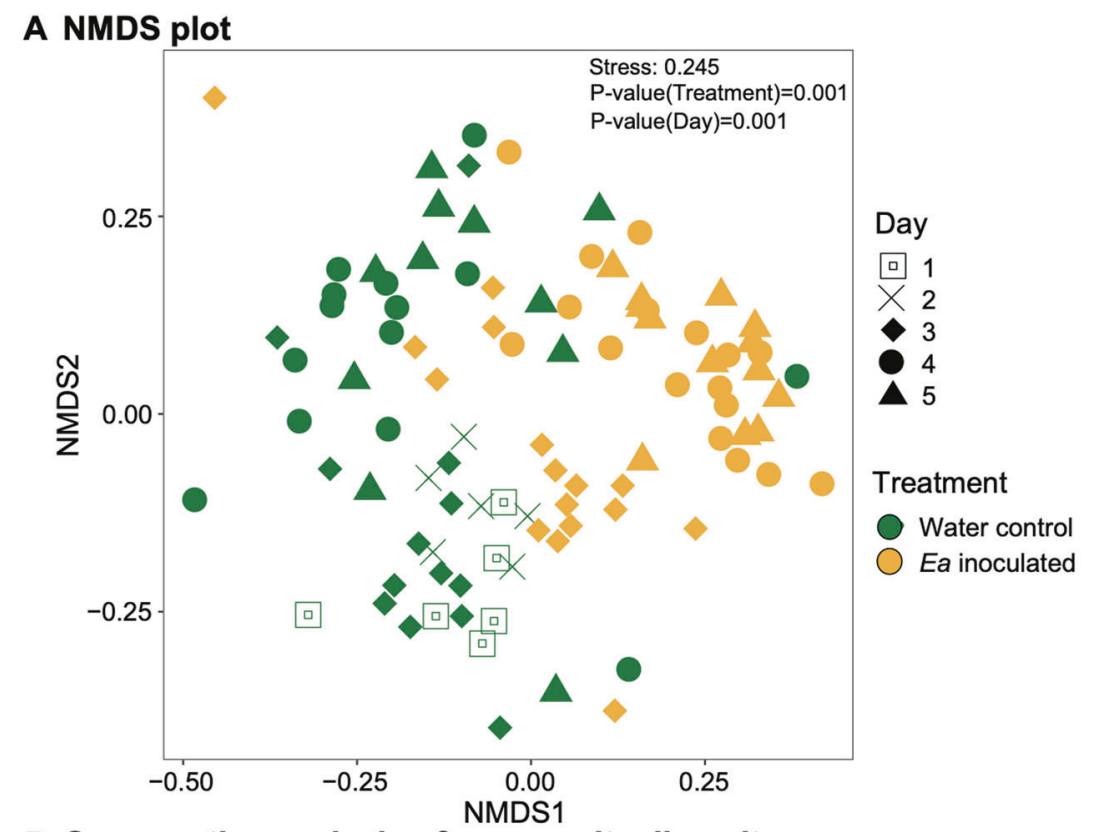

B Comparative analysis of community diversity

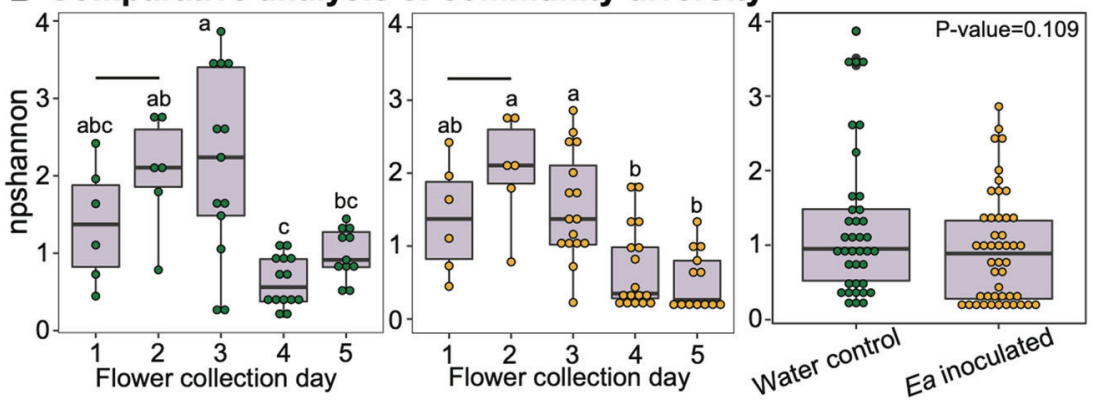

Fig. 4 Non-metric multidimensional scaling (NMDS) plot displaying relationships of stigma microbial community composition. Samples from water control samples are shown in green and $\boldsymbol{E a}$ inoculated samples are shown in gold. a Non-metric multidimensional scaling (NMDS) plot displaying relationships of stigma microbial community composition in samples from water control (green) and $E a$ inoculated samples (gold). Symbols indicate stigma sample collection day. The distances were determined using the Bray-Curtis metric and the stress value of the ordination is indicated. Statistically significant differences in clustering were evaluated via the Adonis permutation test and $P$ values are indicated. b Comparative analysis of community diversity (Shannon index) among stigma samples. Changes in diversity over time for the water control samples (left panel) and the $E a$-inoculated samples (middle panel), respectively. The bar above day 1 and day 2 indicates the pre-treatment samples, which are the same between the panels. Overall diversity of water control samples versus $E a$-inoculated samples (far right panel). Statistically significant differences were identified by ANOVA comparisons of means, employing a post hoc Tukey-Kramer test for multiple comparisons. Boxes labeled with different letters showed statistically significant differences $(P$ value $<0.05)$. 
Fig. 5 a DNA copy numbers of the $E a$ specific gene ams $C$ ordered by abundance in 100 flowers. The dashed line represents average copy number across the samples. b Relative abundance (\%) of the two major bacterial families within Proteobacteria in the stigma microbiome of 100 flowers. Each column represents an individual flower. The columns are ordered by ams $C$ copy number to match (a). c OTUs within the family

Pseudomonadaceae and d Enterobacteriaceae. The category "rare" represents the sum of the remaining taxa.

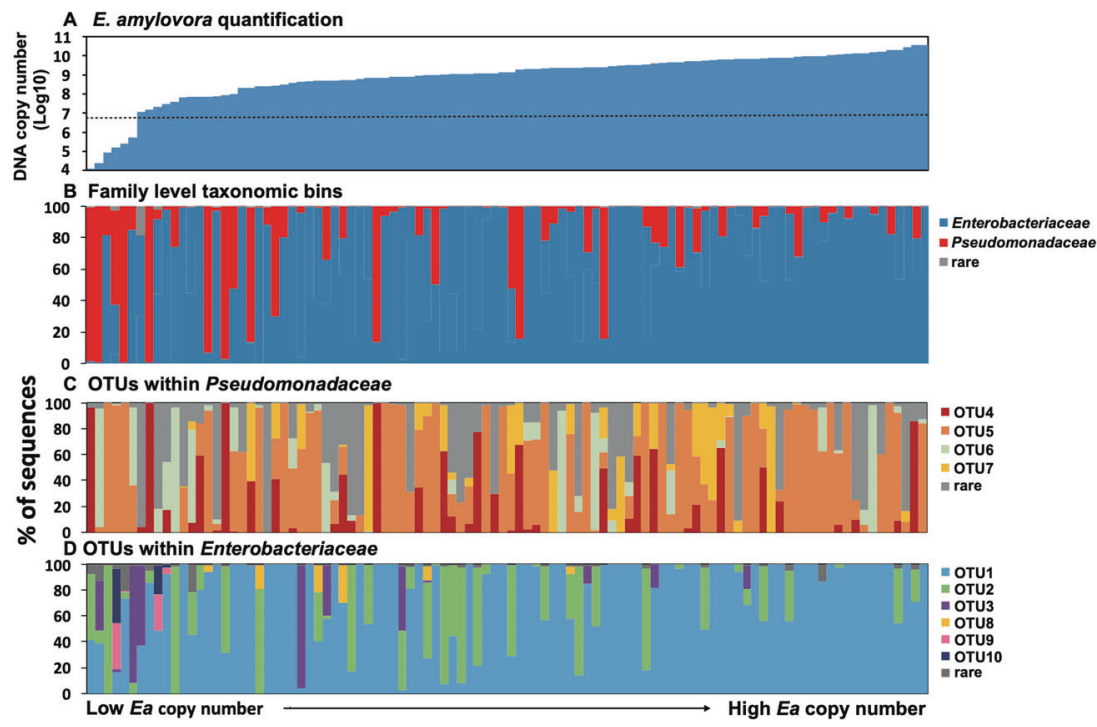

confirmed by permutational multivariate ANOVA $(P<$ 0.001 , Fig. 4a). In addition, samples were also clustered based on days post-bloom $(P<0.001$, Fig. 4a). Diversity of the stigma communities was assessed by calculating the Shannon's Diversity index. For both control and Eainoculated datasets there was a trend toward increased diversity in the early time points, which then decreased by days 4 and 5 (Fig. 4b, $P<0.05$ ). When the control and $E a$ inoculated datasets were combined to test the overall effect of pathogen presence on microbial diversity, there was no significant difference in diversity due to $E a$ treatment (Fig. $4 b, F_{1,82}=2.788, P=0.109$ ).

Collectively, these findings indicate that taxonomically diverse microbial populations initially colonize the stigma of the apple flower. Gradually, a community dominated by representatives of the Pseudomonadaceae and Enterobacteriaceae families outcompetes these populations and become the predominant community members (Fig. 2), which results in an overall decrease in diversity of the stigma microbial community (Fig. 4b, c). In the face of $E a$ challenge there is a significant shift in the composition of the microbial community (Fig. 4a). Yet, there is no significant effect on the diversity of the community as a whole in comparison to the control flowers (Fig. 4b).

\section{The influence of $\mathrm{Ea}$ inoculation on 100 spatially separated flower clusters}

Having shown that inoculation of $E a$ influenced the development and composition of the stigma microbiome, we wanted to expand this analysis to include a larger sample size, encompassing the spatial variation in microbiome composition between flowers and to link microbiome composition to disease development. In this effort, 100 spatially separated flower clusters ( 400 individual flowers) were inoculated with $E a$. Stigmas from one individual flowers were collected from each flower cluster for microbiome characterization, while the remainder of the flowers were left on the tree to assess the rate of disease development (Fig. 1, Fig. S1). Three weeks after $E a$ inoculation, only $42.4 \%$ of the remaining flowers developed fire blight symptoms. The variation in disease development extended to flower clusters, with diseased and healthy flowers coexisting in the same cluster (Fig. 1). Given that the genetic background of the host, flower age, and pathogen exposure were all identical between the inoculated flowers, and the trials were all performed in the same orchard and thus under the same environmental conditions, these observations suggest that none of these factors are sufficient to explain or predict disease occurrence at the single flower level.

\section{Variation in genome copies of Ea among the $\mathbf{1 0 0}$ flower clusters}

We measured the ams $C$ copy number from 100 individual flowers by qPCR. The copy number varied from $1.3 \times 10^{4}$ to $3.7 \times 10^{10}$. The average was $4.4 \times 10^{9}$ (dashed line, Fig. 5a) and the majority $(90 \%)$ of stigmas harbored $>3.0 \times$ $10^{7}$ gene copies of $E a$, which is similar to the average of day 4 inoculated flowers in the temporal dynamics study. This data suggest that the majority of the stigmas have surpassed the threshold population of $\mathrm{ca} 10^{5}-10^{7}$ cells that are thought to be required for disease development $[24,25]$.

\section{Microbiome composition among the 100 flower clusters}

A total of $4,176,840$ high-quality $16 \mathrm{~S}$ rRNA gene sequences were recovered from the 100 flowers, with the number 
Fig. 6 Correlation between $E a$ copy numbers and (a) relative abundance (\%) of OTU1. b Relative abundance $(\%)$ of sequences within the Pseudomonadaceae family (c) community diversity (Shannon index) and $\mathbf{d}$ number of recovered OTUs. Correlations were determined with AVONA test of linear effects model with Shannon diversity and recovered OTUs, and exponential effects model with OTU1 and Pseudomonadaceae-related sequences. The dashed line is the threshold population $\left(10^{7}\right.$ cells) that are thought to be required for disease development.
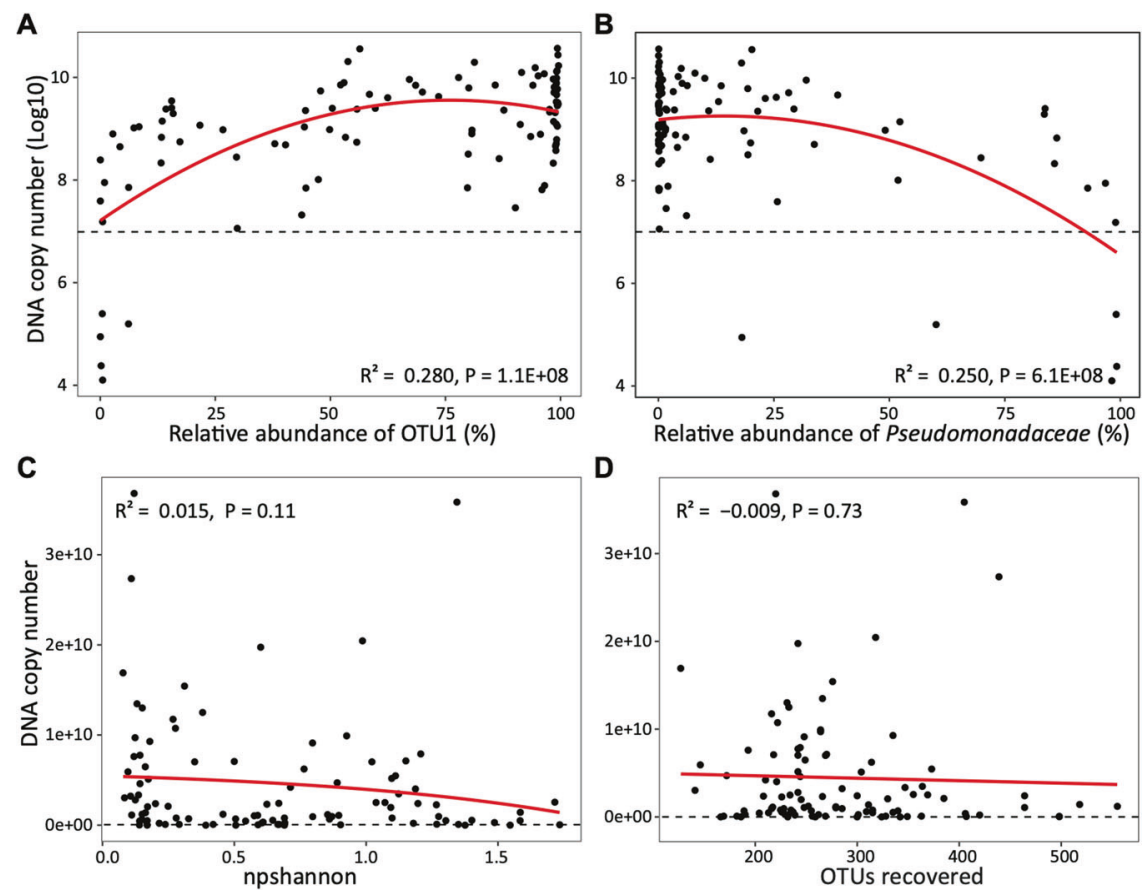

of sequences ranging from 19,297 to 80,130 per sample. After normalizing sampling to the smallest dataset, clustering produced 27,843 OTUs (mean 282 per sample) at $100 \%$ sequence similarity. The detailed information for each dataset is presented in Table S2.

At the phylum level, 22 phyla were identified among the sequences. The most abundant, Proteobacteria, ranged from $96.8 \%$ to $100 \%$ of recovered sequences, followed by Actinobacteria (0-0.5\%), Cyanobacteria (0-1.6\%), and Firmicutes (0-1.5\%) (Fig. S3). Within the Proteobacteria, 59 families were identified and Pseudomonadaceae (red bar in Fig. 5b) and Enterobacteriaceae (blue bar) were predominant ( $>81.5 \%$ in each sample). Notably, the proportion of Pseudomonadaceae and Enterobacteriaceae varied widely among the 100 samples, from $0.02 \%$ to $99.20 \%$ and from $0.45 \%$ to $99.97 \%$, respectively (Fig. 5 b).

\section{OTUs within the Pseudomonadaceae and Enterobacteriaceae}

Sequences within Pseudomonadaceae and Enterobacteriaceae were classified to deeper taxonomic ranks to investigate if particular OTUs were associated with $E a$ abundance. Of the ten most abundant OTUs in the dataset, four belonged to the Pseudomonadaceae and six to the Enterobacteriaceae, representing five different genera (Table S3). By and large each flower harbored a unique microbiome composition, with widely varying abundance of the predominant OTUs among the samples (Fig. 5c, d). Furthermore, there was no observable pattern in specific OTUs being co-abundant in the samples with a low abundance of $E a$ (Fig. 5b, c). Correlational analysis confirmed these observational data, with no significant correlations between the relative abundance of the numerically dominant Pseudomonadaceae OTUs and Ea absolute abundance (Fig. S4A) and similarly low explanatory power for the Enterobacteriaceae OTUs (Fig. S4B).

\section{Correlates of $\mathrm{Ea}$ abundance to metrics of the stigma microbiome}

To test if there were any aspects in the community data that were predictive of $E a$ abundance we performed four correlational analyses. First, the most abundant OTU in the dataset (OTU1) shared $100 \%$ sequence identity with the inoculated Ea strain (Table S3). Therefore, we tested the correlation between the relative abundance of OTU1 and the ams $C$ gene copy number of $E a$, and thereby testing if the relative abundance of OTU1 was correlated to $E a$ absolute abundance (Fig. 6a). The result showed that there was a significant positive relationship between the two metrics $(P<0.001)$, but with an $R^{2}=0.280$, suggesting low explanatory power. This low explanatory power could be due to two factors. First, the relative abundance of OTU1 is an imperfect measure of $E a$ abundance, as there are likely other members of the microbiome that are closely related to $E a$ that cluster into the same OTU. Second, as relative abundance is a proportional value, it is bounded at the upper end at $100 \%$ of recovered sequences. Thus, as Ea absolute abundance increases, eventually proportional abundances can no longer increase. As can be seen in Fig. 5a several stigmas with similar $E a$ counts showed wide variations in 
sequences belonging to OTU1 (Fig. 6a). Thus, these data suggest that increasing $E a$ counts will generally inflate the recovery of $E a$ OTUs, but there is little predictive power in estimating absolute pathogen abundance from relative abundance data. Similarly, we investigated if there was a predictive relationship between the relative abundance of the Pseudomonadaceae and the copy numbers of Ea. In this way we could test if the presence of the Pseudomonadaceae may inhibit $E a$ growth. The relationship displayed a significant negative pattern $(P<0.001)$ but again had a low predictive value $\left(R^{2}=0.250\right.$, Fig. $\left.6 \mathrm{~b}\right)$. Thus, the proportion of recovered Pseudomonadaceae sequences also showed poor predictive value in determining pathogen abundance. Finally, we tested if $E a$ abundance was correlated to two different metrics of diversity of the stigma microbiome, Shannon's diversity index and the number of recovered OTUs. In both cases there was no relationship between $E a$ abundance and diversity (Fig. 6c, d). Thus, there was no apparent effect of $E a$ abundance on the overall diversity of the stigma microbial communities.

\section{Discussion}

The apple flower microbiome has been previously recognized as an important factor for plant health and as a potential source of biocontrol agents against plant pathogens $[1,10,26]$. In addition, since the stigma is the major site of pollination and supports the growth of a large microbial population, microbial growth on the stigma may also influence pollination [27, 28]. Thus, the stigma of a flower is a particularly important plant tissue for studying the microflora that associate with plants. Yet, information concerning the establishment, composition, and development of the microbiome on flower stigmas, as well as the disturbance by the colonization of a phytopathogen, are largely lacking. Previous studies have generally described the flower microbiome from whole flowers or nectar $[1,2,29]$. Our previous work has shown that the stigma generally harbors an overlapping set of OTUs as other flower parts including the stamen, petals, and receptacle, although particular OTUs tend to be overrepresented in the stigma [7]. In this regard, the stigma is likely a representative model of the overall flower microbiome. In this study, we present data based on collecting the stigmas from a single flower, increasing both the temporal and spatial resolution at which the microbiome can be characterized.

Temporal dynamics are important for understanding the evolution of microbial communities [30-32]. Shade et al. characterized the development of the microbiome on pools of apple flowers under a management program of treating the flowers with the antibiotic streptomycin to control fire blight. They found bacteria in the phyla TM7 and Deinococcus were predominant and showed signals of ecological successions with flower age [2]. We show that bacteria within the families Pseudomonadaceae and Enterobacteriaceae were numerically dominant (Fig. 2), which is more congruous with other studies of both the culture-dependent [10] and culture-independent characterizations [7] of apple flower microbial populations. The discrepancy between this study and the [2] report is likely due to methodological differences between studies or PCR biases induced by different PCR primer and blocking pairs versus the PNA clamps used here. In either case, both studies identified strong signals in how the microbiome is structured with flower age. The data presented here point to a core microbiome that was gradually established on the stigma predominantly composed of Pseudomonadaceae and/or Enterobacteriaceae within the phylum Proteobacteria (Fig. 2). The succession of these families was associated with a reduction of other bacterial taxa, such as the Moraxellaceae, Xanthomonadaceae, and Burkholderiaceae, which were only present in the early stages of bloom (Fig. 2). Concurrently, the later stages of bloom were associated with a lower diversity, supporting the observation that a small number of taxa had monopolized the stigma environment as the flower aged (Fig. 2). These observations are consistent with the stigmas being open to colonization by numerous bacteria in the initial stages of bloom. As the petals open, multiple bacteria carried by wind, dew, or insects are introduced to the stigma creating a diverse microbial population. However, with time those bacteria best adapted to the stigma environment prevail and flourish. Yet this suggests that environmental conditions at the time of petal opening may have a large impact on the establishment and development of the stigma microbiome. As the stigmas collected here were all present on flowers opened on the same date, the temporal dynamics are somewhat confounded with environmental conditions. Thus, the community succession dynamics described here only represent a single possible trajectory for the development of the microbiome. Increasing the number of temporal studies performed in different regions and under varied climatic conditions will better constrain the factors that govern the assembly the stigma microbiome. However, the dominance of just two bacterial families on the stigma is analogous to other observations, that complex microbial communities inoculated into a simple medium converge on a state similarly composed of bacteria in the families Pseudomonadaceae and Enterobacteriaceae, a phenomenon referred to as "emergent simplicity" [33]. Thus, there may be conserved rules that govern the assembly of microbial communities, with respect to niche adaptation [5, 6], and microbial competition [34]. Yet, predicting specific microbiome states of individuals or whether the 
factors that govern community assembly are deterministic or stochastic still remain significant knowledge gaps.

Inoculation of the flowers with $E a$ induced a significant shift in the structure of the microbiome (Fig. 4a), but the data indicated that the abundance of $E a$ did not alter microbiome diversity (Fig. 6c, d), but $E a$ abundance was negatively correlated with the presence of other microbes, particularly within the family Pseudomonadaceae (Fig. 6b), although the relationship showed little predictive value in linking Pseudomonadaceae relative abundance to absolute $E a$ abundance. Most notably $90 \%$ of inoculated flowers demonstrated large counts of $E a\left(>3.0 \times 10^{7}\right.$ pathogen specific gene copies) and a high relative abundance of sequences identical to the inoculated pathogen (Fig. 5a, d), yet less than half of the flowers (42\%) later developed fire blight symptoms. As the stigma sampling for microbiome characterization is by necessity destructive, we cannot definitively link the status of the microbiome to disease development. However, these data strongly point to the absolute abundance of $E a$ to be a poor predictive measurement of disease occurrence [24]. Thus, there must be another bottleneck in fire blight disease development beyond $E a$ growth on the stigma. These could include microclimate [35], antimicrobial compounds or yeasts in the nectar [10, 29], and host system sensing signals for detecting high bacterial density [36]. Yet, the observation of a high carrier rate of a pathogen with low disease incidence is synonymous with reports for many human pathogens. For instance, it is well established that $20-40 \%$ of the population are asymptomatic persistent carriers of Staphylococcus aureus, with a further 70-90\% of people considered transient carriers [37]. Yet only a minority of people will develop diseases such as sepsis, pneumonia, or osteomyelitis caused by $S$. auerus infection [38, 39]. A similar phenomena are observed for Cutibacterium acnes as a contributor to skin acne, which is also a major population in the healthy skin microbiome [40]. In this respect, the dynamics of $E a$ growth and fire blight development appear to follow similar dynamics of other diseases, with a high carrier rate, but lower disease incidence. The consequences of these potentially high carrier rates of $E a$ on the incidence rate of fire blight disease are currently unknown. For instance, it is possible that asymptomatic flowers may act as an unrecognized reservoir of the pathogen.

\section{Conclusion}

In this study, we show that the apple flower stigma microbiome shares many characteristics with other host microbiome systems. In the initial stages of stigma colonization, the microbiome is temporally dynamic, which eventually settles into an equilibrium community (Fig. 2).
Similar dynamics have been found in infants, fish, and soil [41-43]. At the OTU level, individual flowers harbor largely unique microbiomes (Fig. $5 \mathrm{c}$, d), similar to vertebrates and insects [44-46]. Despite the diversity of the stigma microbiome at the OTU level ( 200 OTUs per sample), the OTUs fell into just two predominant families (Pseudomonadaceae and Enterobacteriaceae) that differed in abundance between individual flowers (Figs. 2 and 5). This mirrors the observation of the dominance of the Firmicutes and Bacteroidetes in the human intestinal tract, the so-called Firmicutes/Bacteroidetes ratio, and its potential influence on characteristics such as obesity [47, 48]. Finally, we observe that virtually all flowers exposed to the phytopathogen $E a$ developed large pathogen loads (Fig. 5), yet only a fraction $(\sim 42 \%)$ of the flowers developed disease, reflecting a common observation that pathogen burden is not always predictive of disease development [36, 49]. This suggests that to the extent that natural microbiome members may be acting as biocontrol agents they are likely acting through regulating pathogen activity rather than abundance. This highlights the importance of characterizing bacterial interactions and functional ecology of the stigma microbiome. Thus, we propose that the stigma microbiome represents a model system that can be employed to investigate the rules that govern microbial community assembly, development, and disease progression and severity.

\section{Data availability}

The sequences data, including negative controls, are deposited at the Sequence Read Archive under accession number PRJNA597302.

Acknowledgements We thank Sali Diallo and Zach Seltzer for their technical support. This study was supported by the USDA-NIFAOrganic Transitions grant 2017-51106-27001, USDA-NIFA-Agricultural Microbiome grant 2020-67013-31794, Northeastern IPM Center partnership grant and USDA-Specialty Crop Block Grant (SCBG) through the Department of Agriculture, State of Connecticut.

\section{Compliance with ethical standards}

Conflict of interest The authors declare that they have no conflict of interest.

Publisher's note Springer Nature remains neutral with regard to jurisdictional claims in published maps and institutional affiliations.

\section{References}

1. Aleklett K, Hart M, Shade A. The microbial ecology of flowers: an emerging frontier in phyllosphere research. Botany. 2014;92: 253-66.

2. Shade A, McManus PS, Handelsman J. Unexpected diversity during community succession in the apple flower microbiome. MBio. 2013;4:e0602-12. 
3. Ambika Manirajan B, Ratering S, Rusch V, Schwiertz A, Geissler-Plaum R, Cardinale M, et al. Bacterial microbiota associated with flower pollen is influenced by pollination type, and shows a high degree of diversity and species-specificity. Environ Microbiol. 2016;18:5161-74.

4. Tucker CM, Fukami T. Environmental variability counteracts priority effects to facilitate species coexistence: evidence from nectar microbes. Proc R Soc B: Biol Sci. 2014;281:20132637.

5. Pusey PL, Rudell DR, Curry EA, Mattheis JP. Characterization of stigma exudates in aqueous extracts from apple and pear flowers. HortScience. 2008;43:1471-8.

6. Stockwell V, McLaughlin R, Henkels M, Loper J, Sugar D, Roberts R. Epiphytic colonization of pear stigmas and hypanthia by bacteria during primary bloom. Phytopathology. 1999;89: $1162-8$.

7. Steven B, Huntley RB, Zeng Q. The influence of flower anatomy and apple cultivar on the apple flower phytobiome. Phytobiomes. 2018;2:171-9.

8. Norelli JL, Jones AL, Aldwinckle HS. Fire blight management in the twenty-first century: using new technologies that enhance host resistance in apple. Plant Dis. 2003;87:756-65.

9. Thomson S, Wagner A, Gouk S, editors. Rapid epiphytic colonization of apple flowers and the role of insects and rain. VIII International Workshop on Fire Blight. vol 489. ISHS Acta Horticulturae; Kusadasi, Turkey. 1998.

10. Pusey PL, Stockwell VO, Mazzola M. Epiphytic bacteria and yeasts on apple blossoms and their potential as antagonists of Erwinia amylovora. Phytopathology. 2009;99:571-81.

11. Sinclair L, Osman OA, Bertilsson S, Eiler A. Microbial community composition and diversity via $16 \mathrm{~S}$ rRNA gene amplicons: evaluating the illumina platform. PLoS ONE. 2015;10:e0116955.

12. Pirc M, Ravnikar M, Tomlinson J, Dreo T. Improved fireblight diagnostics using quantitative real-time PCR detection of Erwinia amylovora chromosomal DNA. Plant Pathol. 2009;58:872-81.

13. Cui Z, Yuan X, Yang C-H, Huntley RB, Sun W, Wang J, et al. Development of a method to monitor gene expression in single bacterial cells during the interaction with plants and use to study the expression of the type III secretion system in single cells of Dickeya dadantii in potato. Front Microbiol. 2018;9:1429.

14. Schloss PD, W S, Ryabin T, Hall JR, Hartmann M, Hollister EB, et al. Introducing mothur: open-source, platform-independent, community-supported software for describing and comparing microbial communities. Appl Environ Microbiol. 2009;75:7537-41.

15. Rognes T, F T, Nichols B, Quince C, Mahé F. VSEARCH: a versatile open source tool for metagenomics. PeerJ. 2016;4:e2584.

16. Westcott SL, S P. OptiClust, an improved method for assigning amplicon-based sequence data to operational taxonomic units. MSphere. 2017;2:e00073-17.

17. Quast C, P E, Yilmaz P, Gerken J, Schweer T, Yarza P, et al. The SILVA ribosomal RNA gene database project: improved data processing and web-based tools. Nucleic Acids Res. 2012;41: D590-D6

18. Wang Q, Garrity GM, Tiedje JM, Cole JR. Naive Bayesian classifier for rapid assignment of rRNA sequences into the new bacterial taxonomy. Appl Environ Microbiol. 2007;73:5261-7.

19. Dixon P. VEGAN, a package of $\mathrm{R}$ functions for community ecology. J Vegetation Sci. 2003;14:927-30.

20. Wickham H. ggplot2: elegant graphics for data analysis. Springer; New York. 2016.

21. Palacio-Bielsa A, R M, Llop P, López MM. Erwinia spp. from pome fruit trees: similarities and differences among pathogenic and non-pathogenic species. Trees. 2012;26:13-29.

22. Kube M, M A, Müller I, Kuhl H, Beck A, Reinhardt R, Geider K. The genome of Erwinia tasmaniensis strain Et1/99, a nonpathogenic bacterium in the genus Erwinia. Environ Microbiol. 2008;10:2211-22.
23. Geider K, A G, Du Z, Jakovljevic V, Jock S, Völksch B. Erwinia tasmaniensis sp. nov., a non-phytopathogenic bacterium from apple and pear trees. Int J Syst Evolut Microbiol. 2006;56: 2937-43.

24. Thomson S. The role of the stigma in fire blight infections. Phytopathology. 1986;76:476-82.

25. Johnson KB, S V. Management of fire blight: a case study in microbial ecology. Annu Rev Phytopathol. 1998;36:227-48.

26. Berendsen RL, Pieterse CM, Bakker PA. The rhizosphere microbiome and plant health. Trends Plant Sci. 2012;17:478-86.

27. Albrecht M, Padrón B, Bartomeus I, Traveset A. Consequences of plant invasions on compartmentalization and species' roles in plant-pollinator networks. Proc R Soc B: Biol Sci. 2014;281: 20140773.

28. Edlund AF, Swanson R, Preuss D. Pollen and stigma structure and function: the role of diversity in pollination. Plant Cell. 2004;16: S84-S97.

29. Fridman S, Izhaki I, Gerchman Y, Halpern M. Bacterial communities in floral nectar. Environ Microbiol Rep. 2012;4:97-104.

30. Yuan J, Chaparro JM, Manter DK, Zhang R, Vivanco JM, Shen Q. Roots from distinct plant developmental stages are capable of rapidly selecting their own microbiome without the influence of environmental and soil edaphic factors. Soil Biol Biochem. 2015;89:206-9.

31. Marschner P, Neumann G, Kania A, Weiskopf L, Lieberei R. Spatial and temporal dynamics of the microbial community structure in the rhizosphere of cluster roots of white lupin (Lupinus albus L.). Plant Soil. 2002;246:167-74.

32. Bardgett RD, Bowman WD, Kaufmann R, Schmidt SK. A temporal approach to linking aboveground and belowground ecology. Trends Ecol Evol. 2005;20:634-41.

33. Goldford JE, Lu N, Bajić D, Estrela S, Tikhonov M, SanchezGorostiaga A, et al. Emergent simplicity in microbial community assembly. Science. 2018;361:469-74.

34. Pusey P, Stockwell V, Reardon C, Smits T, Duffy B. Antibiosis activity of Pantoea agglomerans biocontrol strain E325 against Erwinia amylovora on apple flower stigmas. Phytopathology. 2011;101:1234-41.

35. Herrera CM. Microclimate and individual variation in pollinators: flowering plants are more than their flowers. Ecology. 1995;76: 1516-24.

36. Medzhitov R, Schneider DS, Soares MP. Disease tolerance as a defense strategy. Science. 2012;335:936-41.

37. Hamdan-Partida A, González-García S, de la Rosa García E, Bustos-Martínez J. Community-acquired methicillin-resistant Staphylococcus aureus can persist in the throat. Int $\mathrm{J}$ Med Microbiol. 2018;308:469-75.

38. Peacock SJ, de Silva I, Lowy FD. What determines nasal carriage of Staphylococcus aureus? Trends Microbiol. 2001;9:605-10.

39. Von Eiff C, Becker K, Machka K, Stammer H, Peters G. Nasal carriage as a source of Staphylococcus aureus bacteremia. N Engl J Med. 2001;344:11-6.

40. Paetzold B, Willis JR, de Lima JP, Knödlseder N, Brüggemann H, Quist SR, et al. Skin microbiome modulation induced by probiotic solutions. Microbiome. 2019;7:95.

41. Trosvik P, Stenseth NC, Rudi K. Convergent temporal dynamics of the human infant gut microbiota. ISME J. 2010;4:151.

42. Shenhav L, Furman O, Briscoe L, Thompson M, Silverman JD, Mizrahi I, et al. Modeling the temporal dynamics of the gut microbial community in adults and infants. PLoS Comput Biol. 2019;15:e1006960.

43. Giatsis C, Sipkema D, Smidt H, Verreth J, Verdegem M. The colonization dynamics of the gut microbiota in tilapia larvae. PLoS ONE. 2014;9:e103641.

44. Booijink CC, El-Aidy S, Rajilić-Stojanović M, Heilig HG, Troost FJ, Smidt $\mathrm{H}$, et al. High temporal and inter-individual variation 
detected in the human ileal microbiota. Environ Microbiol. 2010;12:3213-27.

45. Bolnick DI, Snowberg LK, Hirsch PE, Lauber CL, Org E, Parks $\mathrm{B}$, et al. Individual diet has sex-dependent effects on vertebrate gut microbiota. Nat Commun. 2014;5:4500.

46. Colman DR, Toolson EC, Takacs-Vesbach C. Do diet and taxonomy influence insect gut bacterial communities? Mol Ecol. 2012;21:5124-37.
47. Turnbaugh PJ, Ley RE, Mahowald MA, Magrini V, Mardis ER, Gordon JI. An obesity-associated gut microbiome with increased capacity for energy harvest. Nature. 2006;444:1027.

48. Mariat D, Firmesse O, Levenez F, Guimarăes V, Sokol H, Doré J, et al. The Firmicutes/Bacteroidetes ratio of the human microbiota changes with age. BMC Microbiol. 2009;9:123.

49. Råberg L, Sim D, Read AF. Disentangling genetic variation for resistance and tolerance to infectious diseases in animals. Science. $2007 ; 318: 812-4$. 